Supporting Information for

\title{
Self-Driven "Microfiltration" Enabled by Porous Superabsorbent Polymer (PSAP) Beads for Biofluid Specimen Processing and Storage
}

\author{
Wensi Chen, Ting Wang, Zeou Dou, and Xing Xie
}

School of Civil and Environmental Engineering, Georgia Institute of Technology, Atlanta, Georgia 30332, USA.

*Corresponding Author, E-mail: xing.xie@ce.gatech.edu 


\section{Table of Contents}

\section{Experimental Method}

1.1 Chemicals and Reagents

1.2 Preparation and Characterization of Liquid Samples

1.3 Calculation of Recovery or Rejection Efficiency

\section{Optimization for the PSAP bead synthesis}

2.1 Pore Structure Control

\subsection{Swelling Capacity Control}

\section{Additional Figures}

Figure S1. Preparation of the PSAP beads.

Figure S2. SEM images of the PSAP beads.

Figure S3. Swelling behavior of the PSAP beads in the saline medium.

Figure S4. Uniform size and swelling capacity of the PSAP beads.

Figure S5. Schematic of microfiltration treatment and recovery of target species.

Figure S6. Distribution of fluorescent microspheres near the edge of the PSAP beads.

Figure S7. Swelling ratio of the PSAP beads in biofluid media.

Figure S8. SEM images of the PAM beads prepared with different polymerization conditions.

Figure S9. SEM images of the Poly(SA-co-AM) beads with different monomer compositions.

Figure S10. Swelling ratio of the poly(SA-co-AM) beads in DI water.

Figure S11. SEM images of the poly(SA-co-AM) beads with different crosslinking degrees and charge densities.

\section{Additional Tables}

Table S1. Mercury intrusion test of porous PSAP beads prepared with different PEG contents. Table S2. The composition of the synthetic urine. 


\section{Experimental Method}

\subsection{Chemicals and Biomaterials}

Acrylamide (AM, 99\%), sodium acrylate (SA, 97\%), $N, N^{\prime}$-methylenebisacrylamide (MBA, 99\%), poly(ethylene glycol) (PEG, average $\mathrm{M}_{\mathrm{n}}=6000 \mathrm{~g} \mathrm{~mol}^{-1}$ ), ammonium persulfate (APS, 98\%), urea $(99 \%)$, potassium phosphate monobasic $\left(\mathrm{KH}_{2} \mathrm{PO}_{4}, 99.0 \%\right)$, potassium phosphate dibasic $\left(\mathrm{K}_{2} \mathrm{HPO}_{4}, 99.0 \%\right)$, sodium phosphate monobasic $\left(\mathrm{NaH}_{2} \mathrm{PO}_{4}, 99.0 \%\right)$, sodium hydroxide $(\mathrm{NaOH}$, $97.0 \%)$, hydrogen peroxide $\left(\mathrm{H}_{2} \mathrm{O}_{2}, 30 \mathrm{wt} \%\right.$ in water), catalase (lyophilized powder, $2000-5000$ U/mg), and D-(+)-glucose (99.5\%) were purchased from Sigma-Aldrich (St. Louis, MO). Sodium chloride $(\mathrm{NaCl}, 99.0 \%)$, sodium sulfate $\left(\mathrm{Na}_{2} \mathrm{SO}_{4}, 99.0 \%\right)$, potassium chloride $(\mathrm{KCl}, 99.0 \%)$, magnesium chloride $\left(\mathrm{MgCl}_{2}, 99.0 \%\right)$, and calcium chloride $\left(\mathrm{CaCl}_{2}, 99.0 \%\right)$ were obtained from VWR Chemicals BDH (Radnor, PA). All chemicals were used as received without further purification. DI water with a resistivity of $18.2 \mathrm{M} \Omega \mathrm{cm}$ was produced by a Barnstead nanopure water system (Thermo Scientific, Waltham, MA) and used throughout the experiments. Phosphate buffer $(50 \mathrm{mM})$ was prepared by dissolving $\mathrm{K}_{2} \mathrm{HPO}_{4}(30.75 \mathrm{mM})$ and $\mathrm{KH}_{2} \mathrm{PO}_{4}(19.25 \mathrm{mM})$ in DI water, which was subsequently adjusted to a $\mathrm{pH}$ of 7 at room temperature by $1 \mathrm{M} \mathrm{NaOH}$ solution.

Escherichia coli (E. coli, ATCC 10798 and 15597), Bacillus subtilis (B. subtilis, ATCC 6051), and bacteriophage MS2 (ATCC 15597-B1) were obtained from the American type culture collection (ATCC). Luria-Bertani broth (LB broth), Luria-Bertani agar (LB agar), tryptic soy broth (TS broth), tryptic soy agar (TS agar), nutrient broth and nutrient agar were obtained from BD Difco (Franklin Lakes, NJ).

\subsection{Preparation and Characterization of Liquid Samples}

\section{(1) Glucose Samples}

Glucose was dissolved in saline or synthetic urine medium to reach a concentration of 5 $\mathrm{mM}$. Bovine plasma already contained $\sim 5 \mathrm{mM}$ of glucose, and there would be no need for an extra dosage. The glucose concentration was detected by the colorimetric method using EnzyChrom glucose assay kit II (BioAssay Systems, Hayward, CA) ${ }^{1}$. In general, $20 \mu \mathrm{L}$ sample was added to a $1 \mathrm{~mL}$ plastic cuvette containing $80 \mu \mathrm{L}$ of working reagent and $900 \mu \mathrm{L}$ of water, which was mixed well and incubated at room temperature for $20 \mathrm{~min}$. The absorbance of the reaction mixture at 340 $\mathrm{nm}$ was proportional to the glucose concentration in the sample, and the coefficient was obtained 
from the slope of the standard curve. The effective concentration range for this glucose assay was 0.1 to $3 \mathrm{mM}$, and any sample beyond this range required appropriate dilution.

\section{(2) Catalase Samples}

The catalase stock solution was prepared by dissolving catalase powder in saline medium ( $5 \mathrm{mg} \mathrm{mL}^{-1}$ ) and then filtered by $0.2 \mu \mathrm{m}$ syringe filter (VWR International, Radnor, PA) to remove any undissolved particles or potential microorganisms. Then, the catalase stock was diluted 10 times by saline or biofluid media (synthetic urine or bovine plasma) to reach a final activity at $\sim 800 \mathrm{U} \mathrm{mL}^{-1}$. Since catalase exited in red blood cells, bovine blood didn't require a dosage for catalase. The activities of all catalase samples were determined by a standard spectrophotometric $\operatorname{method}^{2}$. Specifically, the catalase sample was first diluted to the desired detection range of $\sim 50$ to $100 \mathrm{U} \mathrm{mL}^{-1}$. Next, $0.1 \mathrm{~mL}$ of the catalase sample was added to a $3 \mathrm{~mL}$ quartz cuvette containing $2.9 \mathrm{~mL}$ of hydrogen peroxide solution $\left(0.04 \mathrm{wt} \%\right.$ of $\mathrm{H}_{2} \mathrm{O}_{2}$ in the phosphate buffer) and mixed immediately by inversion. The decrease in absorbance of the mixture was monitored by a UV-Vis spectrophotometer (Cary 50, Santa Clara, CA) at $240 \mathrm{~nm}$ for $1 \mathrm{~min}$ and analyzed using a linear regression model. Three replicates were performed for each sample. The catalase activity in the $0.1 \mathrm{~mL}$ sample $\left(c, \mathrm{U} \mathrm{mL}^{-1}\right)$ was determined by the reduction rate of absorbance $\left(k, \mathrm{~min}^{-1}\right)$ :

$$
c=-\frac{k \times 30}{1.0 \mu \text { mole } \mathrm{min}^{-1} U^{-1} \times 43.6 \mathrm{M}^{-1} \mathrm{~cm}^{-1} \times 1.0 \mathrm{~cm}} \quad \text { Eq. } 1
$$

in which $1.0 \mu$ mole $\min ^{-1} \mathrm{U}^{-1}$ was the decomposition rate of $\mathrm{H}_{2} \mathrm{O}_{2}$ by catalase, $43.6 \mathrm{M}^{-1} \mathrm{~cm}^{-1}$ was the molar extinction coefficient for $\mathrm{H}_{2} \mathrm{O}_{2}$ at $240 \mathrm{~nm}, 1.0 \mathrm{~cm}$ was the light path, and 30 was the dilution ratio.

\section{(3) Bacteriophage MS2 Samples}

Bacteriophage MS2 was cultured with the host E. coli (ATCC 15597) in a TS broth at $35^{\circ} \mathrm{C}$ for $\sim 6$ hours. The TS broth was subsequently centrifuged at $4000 \mathrm{rpm}$ for $5 \mathrm{~min}$, and the supernatant was filtered through a $0.2 \mu \mathrm{m}$ filter to remove the residual $E$. coli cells. The purified MS2 was diluted and dispersed in saline or biofluid media (synthetic urine or bovine plasma) to

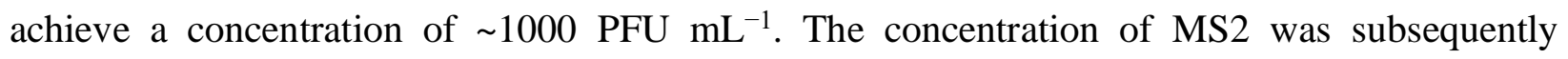
quantified by the double agar layer method with three replicates for each sample ${ }^{3}$.

\section{(4) E. coli Samples}

E. coli cells (ATCC 10798) were cultured in the LB broth to $\log$ phase $\left(35^{\circ} \mathrm{C}\right.$ overnight) and harvested by centrifugation at $4000 \mathrm{rpm}$. After washing with normal saline three times, the $E$. coli suspension was diluted and dispersed in the saline medium to achieve the desired 
concentration of $\sim 500 \mathrm{CFU} \mathrm{mL}^{-1}$. The concentration of live E. coli was measured using a standard spread plating method with three replicates for each measurement ${ }^{4}$.

\subsection{Calculation of Recovery or Rejection Efficiency}

\section{(1) Recovery Efficiency}

The recovery efficiency of the target species (e.g., glucose, catalase, or bacteriophage MS2) was determined by comparing the concentration or activity of the target species released from the PSAP beads $\left(c_{t}\right)$ and in the original liquid sample $\left(c_{0}\right)$. In brief, 10 PSAP beads were applied to treat $1 \mathrm{~mL}$ of the liquid sample. The 10 PSAP beads were taken out until swelling equilibrium achieved and then weighted $(m)$. Next, the hydrated beads in a clean tube were immersed by $2 \mathrm{~mL}$ of added water ( $m_{\text {water }}$ ) and then broken by $5 \mathrm{~s}$ ultrasonication using a probe sonicator (Qsonica Q125, Newtown, CT) at 75\% of amplitude. Thus, the dilution factor (DF) for the target species was calculated:

$$
D F=\frac{m+m_{\text {water }}}{m}
$$

The dilution factor could be adjusted by changing the amount of added water ( $\left.m_{\text {water }}\right)$. After releasing the target species from the hydrated beads, the concentration or activity of the target species in the well-mixed suspension was measured $\left(c_{t}\right)$ to analyze the recovery efficiency for each target species:

$$
\text { Recovery }(\%)=\frac{c_{t}}{c_{0}} \times D F \times 100
$$

For the recovery efficiency calculation, the weight of the dried beads was negligible due to the high swelling ratio of the beads. Another assumption to simply the calculation was the density change during the dilution process was ignored, i.e., the density of the suspension was equal to that of the hydrated beads.

\section{(2) Rejection Efficiency}

The rejection efficiency of the target species (e.g., E. coli or red blood cell) was determined by comparing the concentration of the target species in the residual liquid sample after the treatment $\left(c_{\mathrm{r}}\right)$ and in the original liquid sample $\left(c_{0}\right)$. The operation was similar to the process that determined the recovery efficiency. A total of 10 PSAP beads were applied to treat $1 \mathrm{~mL}$ of liquid sample $\left(V_{0}\right)$, which were subsequently taken out and weighted $(m)$. The volume of the residual liquid sample $\left(V_{\mathrm{r}}\right)$ was calculated: 


$$
V_{r}=V_{0}-m / \rho
$$

Eq. 4

in which the $\rho$ was the density of the hydrated beads (approximately, the density of the biofluid media). Therefore, the rejection efficiency was determined as follows:

$$
\operatorname{Rejection}(\%)=\left(1-\frac{c_{0} V_{0}-c_{r} V_{r}}{\left(V_{0}-V_{r}\right) c_{0}}\right) \times 100
$$




\section{Optimization for the PSAP bead synthesis}

\subsection{Pore Structure Control}

Based on previous studies, we investigate the effects of the polymerization conditions (polymerization temperature, monomer composition, crosslinking degree, and porogen concentration) on the pore structure and swelling behaviors of the resultant PSAP beads. ${ }^{5,6}$ The reaction mixtures containing $10 \%$ of AM, $4 \% \mathrm{MBA}$ and $0.3 \%$ APS were added PEG as the porogen $(5-30 \mathrm{wt} \%)$ and polymerized at three temperatures $\left(70-90^{\circ} \mathrm{C}\right)$ to prepare porous polyacrylamide (PAM) beads. The results shown in Figure S8 indicate that the morphology of the beads is mainly dependent on the PEG content. When the polymerization temperature is $70^{\circ} \mathrm{C}$, no obvious pores can be found in the PAM beads with $5 \mathrm{wt} \%$ of PEG. As the PEG content increases from 5 to $10 \mathrm{wt} \%$, the resultant beads have a few pores, but those pores are small and disconnected. When the PEG content is further increased, both the porosity and the average pore size of the beads increase, and the as-synthesized beads show a uniform pore distribution.

On the other hand, the polymerization temperature is another important factor for phase separation together with the pore structure formation during the polymerization. At the same PEG content (e.g., $10 \mathrm{wt} \%)$, the porosity and the average pore size of the PAM beads increase as the polymerization temperature increases (Figure S8). Nevertheless, although a higher temperature can accelerate and enhance the phase separation between the polymer network and the porogen, a relatively rapid separation rate causes more uneven distribution of pores and the formation of some big pores in the beads. Therefore, $70^{\circ} \mathrm{C}$ was selected as the optimal polymerization temperature for further optimization of the PSAP bead synthesis.

\subsection{Swelling Capacity Control}

The swelling capacity of the polymer depends on the crosslinking degree, charge density, and polymer concentration of the polymer network. ${ }^{7}$ These three factors are related to the concentrations of the crosslinker, the ionic comonomer, and the total monomers in the initial reaction precursor, respectively. Based on the design criteria, the PSAP beads should have a high swelling ability in the water and aqueous solutions, so that the proposed microfiltration treatment of biofluid samples enabled by the PSAP beads is a spontaneous process and does not need additional driving forces. However, regardless of the PEG contents, the swelling ratio of the as- 
prepared porous PAM beads is less than $10 \mathrm{~g} \mathrm{~g}^{-1}$ in DI water, which is not adequate for the demand of super absorbency.

To improve the swelling capacity, an ionic comonomer, SA, was introduced to the reaction system for the production of PSAP beads. The porous poly(SA-co-AM) beads were prepared by precursors containing 2-8 wt\% of SA monomers while the total monomer concentration was fixed at $10 \mathrm{wt} \%$. The addition of ionic monomers changed the precursor properties and polymerization mechanism thus affected the subsequent phase separation and pore generation. As illustrated in Figure S9, when the SA concentration is $2 \mathrm{wt} \%$, the surface of the resulting bead is covered by a dense nonporous polymer layer and numerous extremely small pores distribute inside the bead. As the SA concentration is increased to 4 or $6 \mathrm{wt} \%$, the beads show a uniform porous dendritic structure and the average pore size is between 1 to $2 \mu \mathrm{m}$. If the SA concentration is further increased to $8 \mathrm{wt} \%$, the porosity significantly decreases as well as some particles are embedded on the polymer network. Therefore, a reaction mixture with the monomer ratio (SA/AM) of 4:6 or 6:4 was chosen in the following experiments for further improvement of the bead swelling capacity.

As mentioned, the swelling capacity of the polymer is highly affected by the crosslinking degree. A high crosslinking degree results in the difficulty of water diffusing into the polymer network due to a more compact polymer structure. ${ }^{7}$ Hence porous poly(SA-co-AM) beads with the MBA concentration from 1 to $0.1 \mathrm{wt} \%$ were prepared and characterized. Figure S10 illustrates the weight swelling ratio of the beads at equilibrium in DI water. The results indicate that the crosslinking degree is the dominant factor for the swelling capacity of the beads. As expected, the swelling ratio of the beads increases as the MBA concentration decreases despite the monomer composition. At a high crosslinking degree (0.5-1 wt\% of MBA), there is almost no difference in the swelling ratio for both the precursors. While at a low crosslinking degree $(<0.5 \mathrm{wt} \%$ of MBA), the impact of the charge density in the swelling ratio becomes more distinct. For example, the swelling ratio of the beads prepared by $6 \mathrm{wt} \%$ of SA is $\sim 60 \%$ higher than those prepared by $4 \mathrm{wt} \%$ of SA (117 versus $74 \mathrm{~g} \mathrm{~g}^{-1}$ ) when the MBA concentration is $0.1 \mathrm{wt} \%$. This is because the increase of the ionic group number from the SA monomers results in the increase of counterion concertation inside the polymer network, which contributes to an additional osmotic pressure improving the polymer swelling. ${ }^{8}$

The change of the crosslinking degree also has a slight impact on the pore structure of the resultant beads. As shown in Figure S11, a higher crosslinking degree (i.e., a high MBA 
concentration) results in a more rigid polymer network with more small pores while a lower crosslinking degree (i.e., a low MBA concentration) results in a more relaxed network with a uniform pore distribution. Since the poly(SA-co-AM) beads containing $6 \mathrm{wt} \%$ of SA and $4 \mathrm{wt} \%$ of AM have both a high swelling capacity and a uniform pore distribution, we have selected this composition as the optimal monomer composition for preparing the prospective PSAP beads. 


\section{Additional Figures}

a

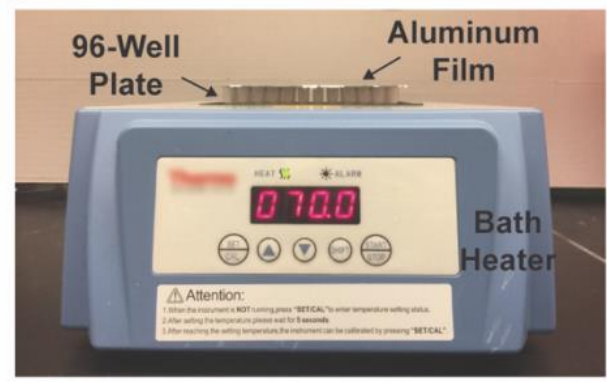

b

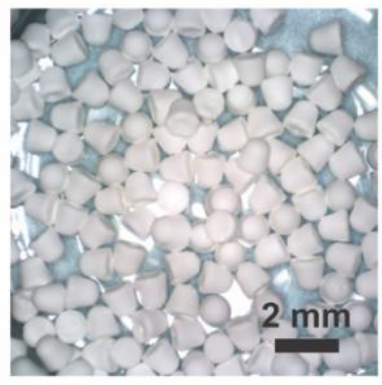

Figure S1. Preparation of the PSAP beads. (a) Experimental setup for the PSAP bead preparation. The 96-well plate was filled by the precursor (15 $\mu \mathrm{L}$ in each well). (b) Optical image of the asprepared PSAP beads.
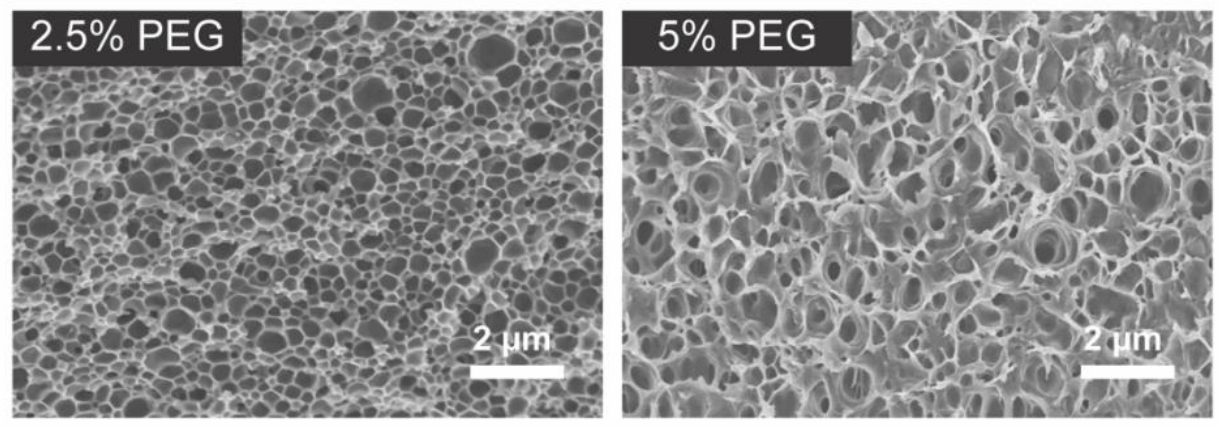

Figure S2. SEM images of the PSAP beads. The beads were polymerized by precursors containing 2.5 or $5 \mathrm{wt} \%$ of PEG. 

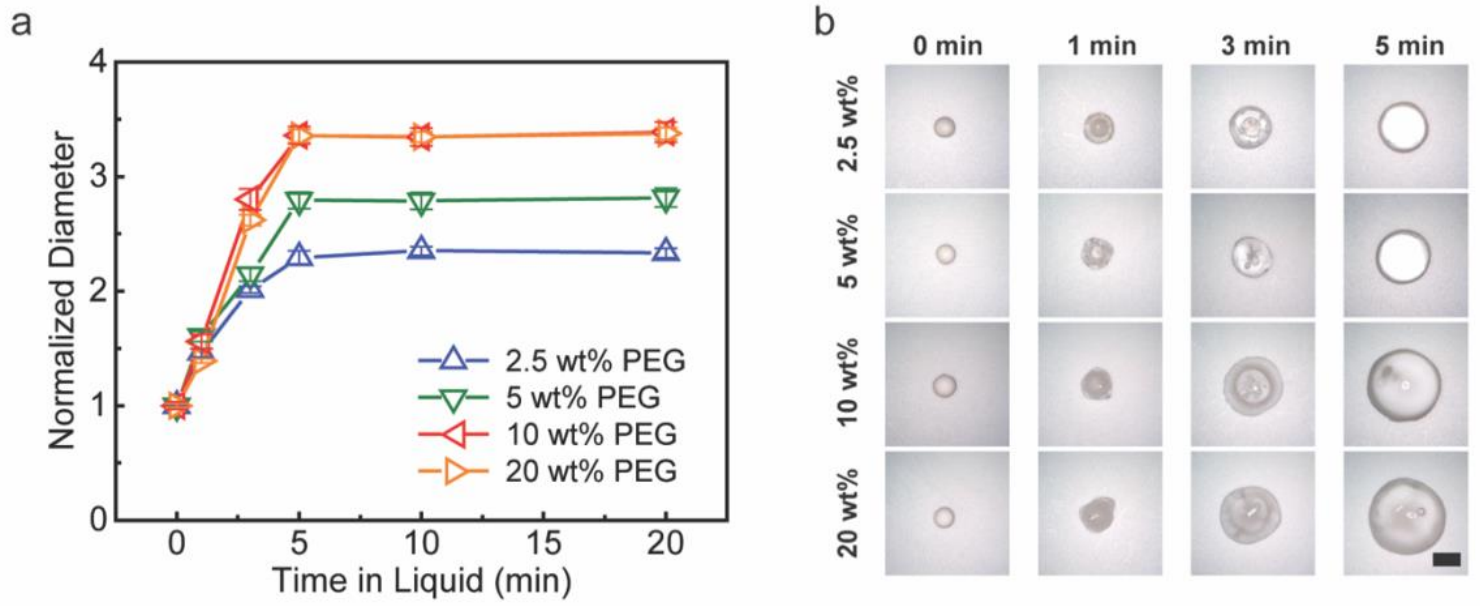

Figure S3. Swelling behavior of the PSAP beads in the saline. (a) The normalized diameter of the PSAP beads prepared with different PEG contents $(2.5,5,10$, and $20 \mathrm{wt} \%)$ in the saline over time. (b) Optical images of the PSAP beads prepared with different PEG contents during the swelling process. The scale bar is $2 \mathrm{~mm}$.


Figure S4. Uniform size and swelling capacity of the PSAP beads. (a) Average weight of a single dry PSAP bead prepared with different PEG contents (10, 15, and $20 \mathrm{wt} \%)$. For each PEG content, 20 of dry PSAP beads were measured. (b) Average weight of a single hydrated PSAP bead after 5 min swelling in $0.9 \% \mathrm{NaCl}$ solution. For each PEG content (10, 15, or $20 \mathrm{wt} \%), 20$ of dry PSAP beads were applied to swelling. 
a

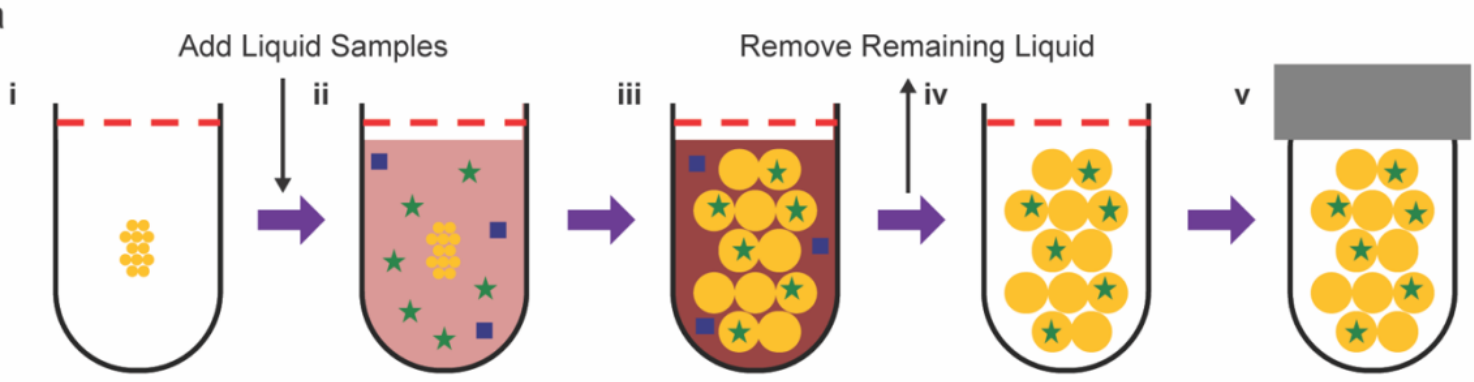

b

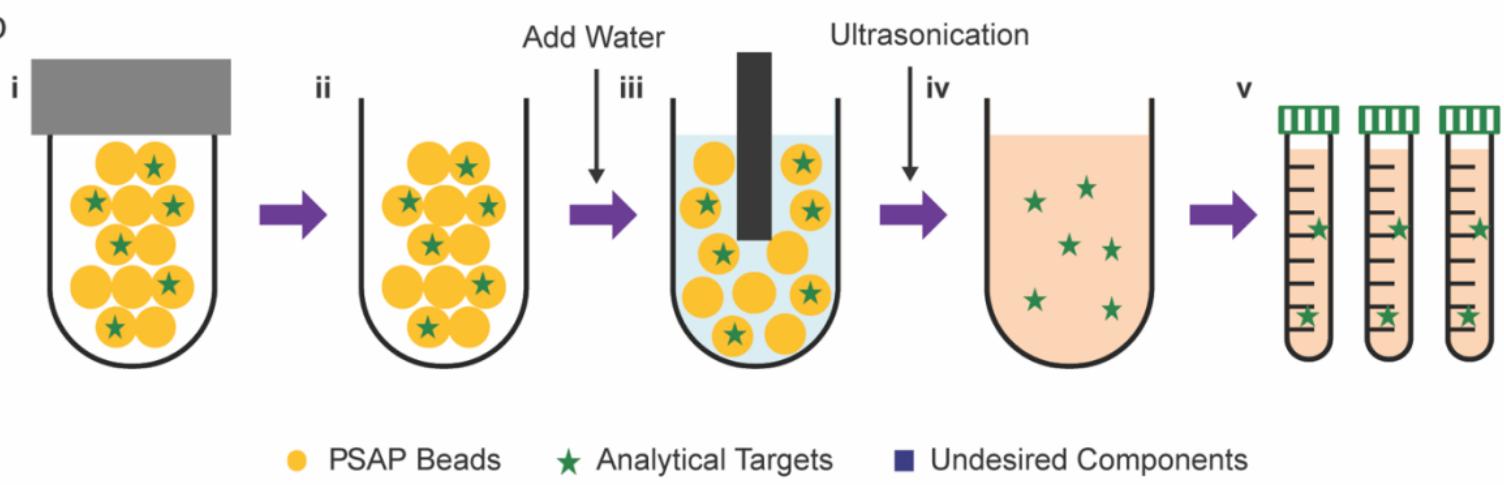

Figure S5. Schematic of microfiltration treatment and recovery of target species. (a) Use PSAP beads to absorb liquid samples and capture analytical targets while excluding undesired components. (b) The analytical targets inside the PSAP beads were released by ultrasonication for further characterization. 


\section{a}

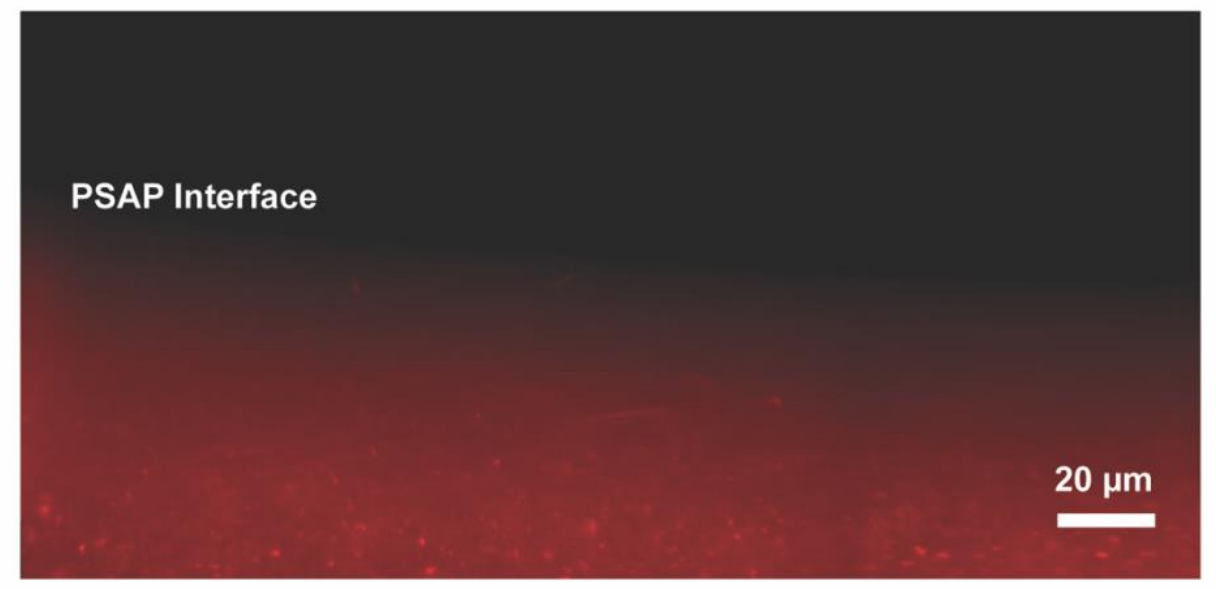

b

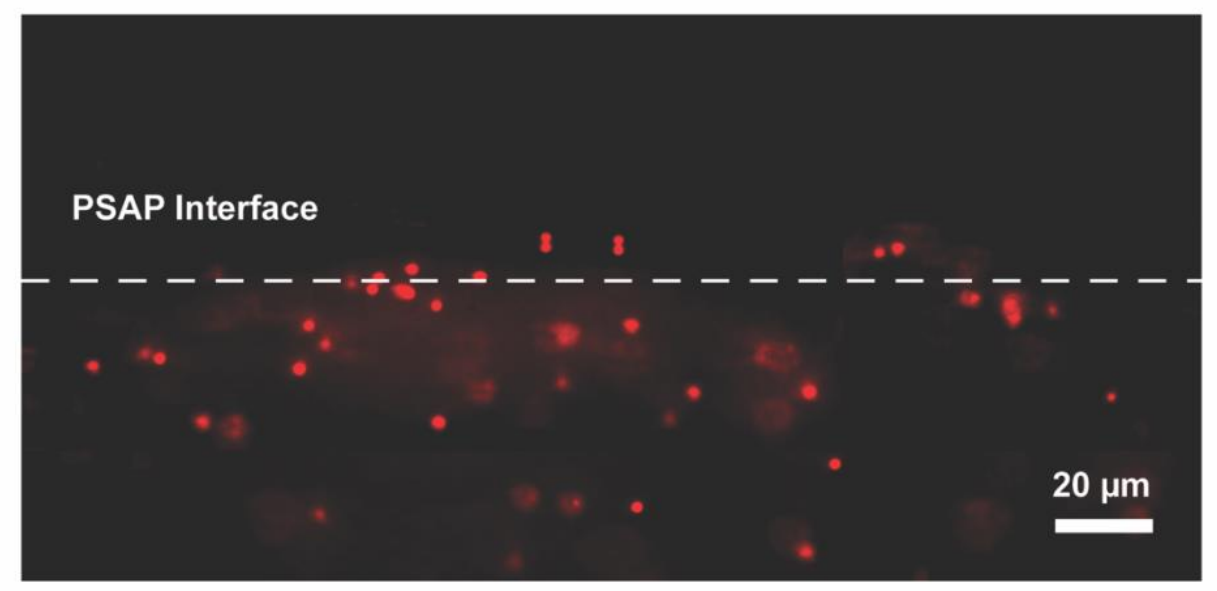

Figure S6. Distribution of fluorescent microspheres near the edge of the PSAP beads after the microfiltration treatment. (a) The small fluorescent target used was $0.1 \mu \mathrm{m}$ in diameter. (b) The large fluorescent target used was $2 \mu \mathrm{m}$ in diameter. 


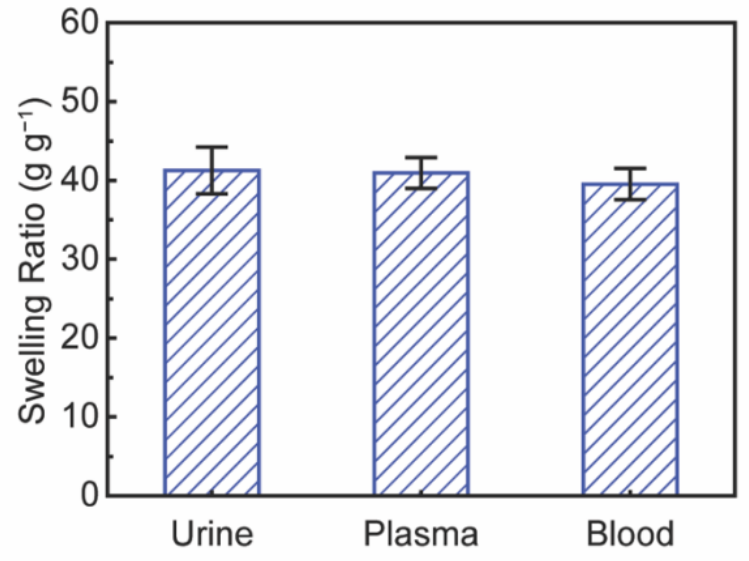

Figure S7. Swelling ratio of the PSAP beads in biofluid media. The PSAP beads were prepared with $10 \mathrm{wt} \%$ of PEG. 


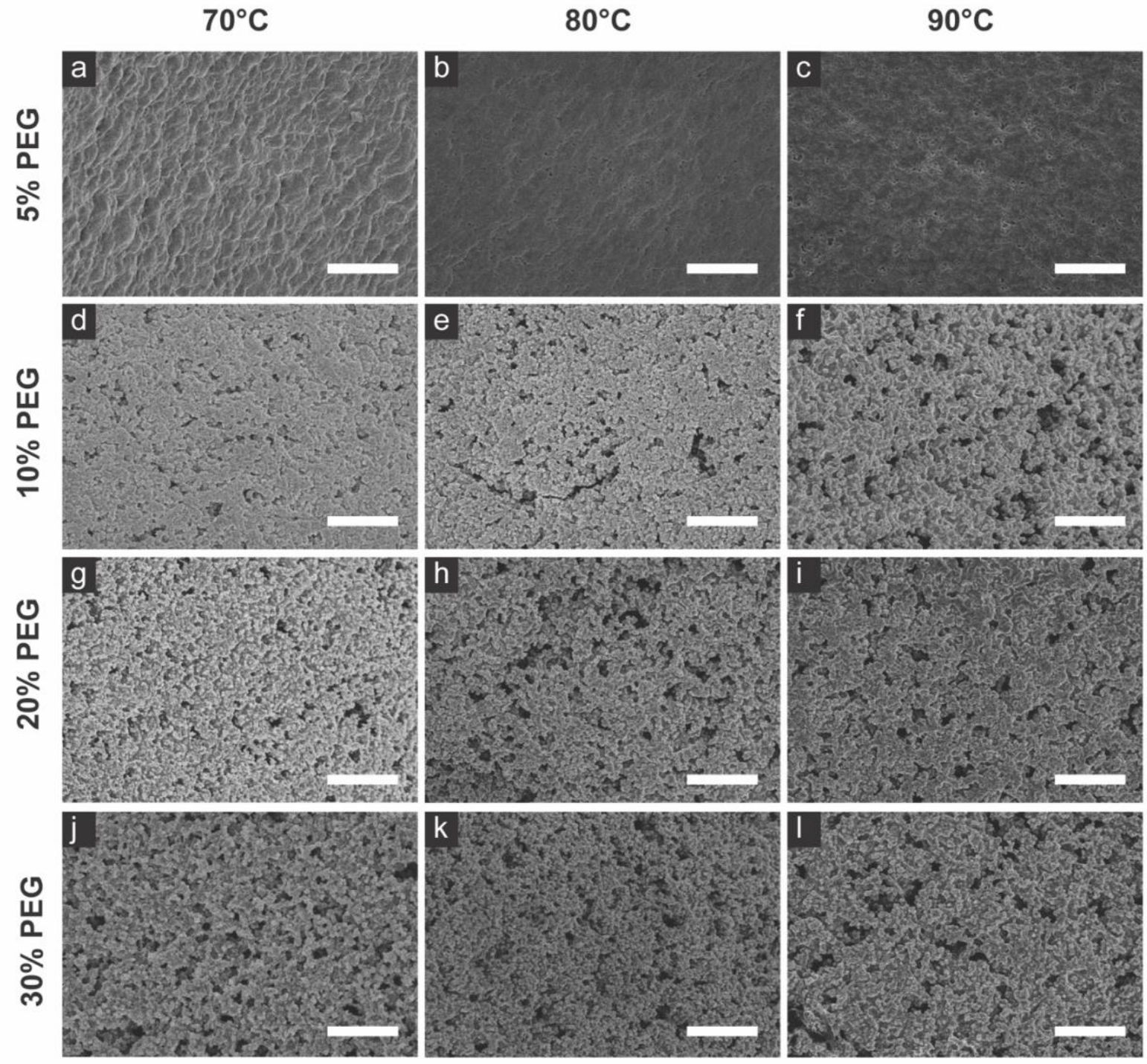

Figure S8. SEM images of the PAM beads prepared with different polymerization conditions. (ac) $5 \mathrm{wt} \%$ of PEG. (d-f) $10 \mathrm{wt} \%$ of PEG. (g-i) $20 \mathrm{wt} \%$ of PEG. (j-1) $30 \mathrm{wt} \%$ of PEG. The three polymerization temperatures were 70,80 and $90^{\circ} \mathrm{C}$, respectively. The AM, MBA, and APS concentrations were maintained constant at 10,4 , and $0.3 \mathrm{wt} \%$, respectively. The reaction time was $15 \mathrm{~min}$ for each condition. The scale bars are all $5 \mu \mathrm{m}$. 

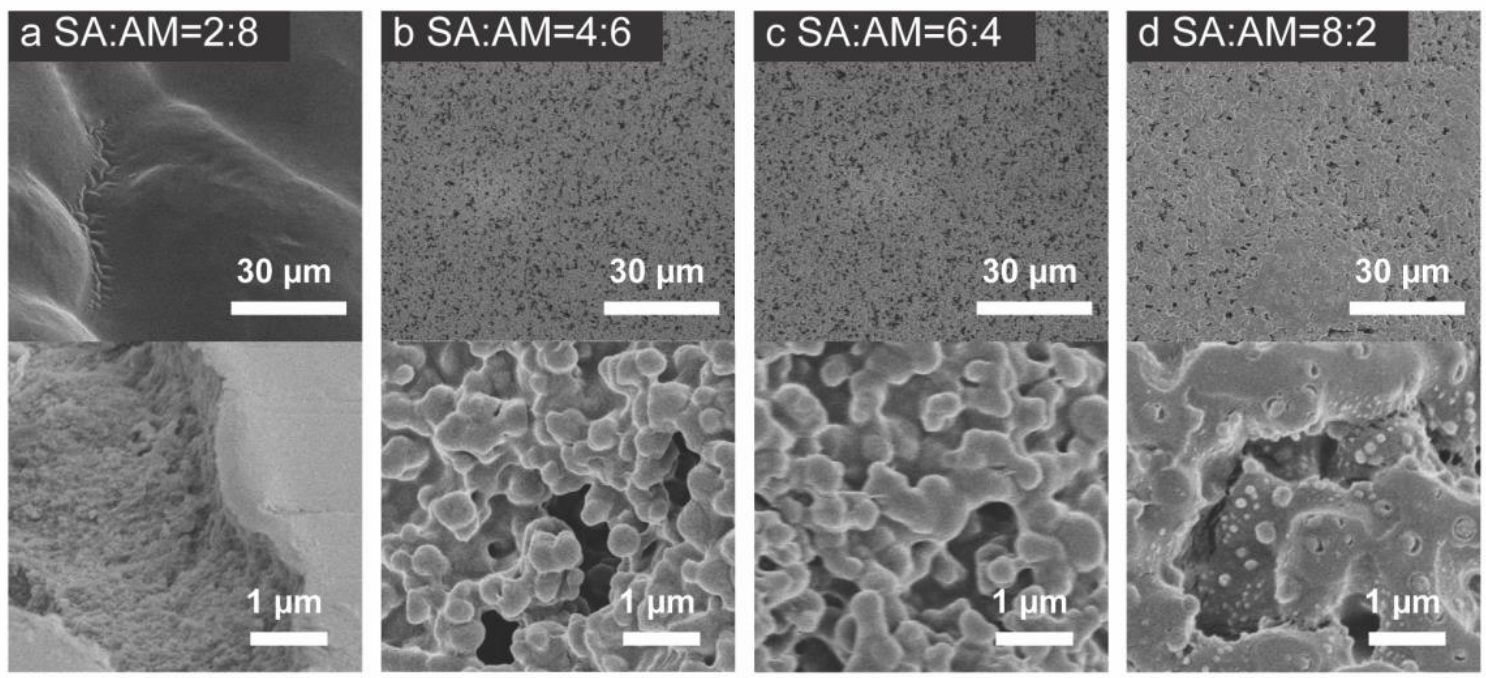

Figure S9. SEM images of the poly(SA-co-AM) beads with different monomer compositions. (a) $S A: A M=2: 8$. (b) $S A: A M=4: 6$. (c) $S A: A M=6: 4$. (d) $S A: A M=8: 2$. The total monomer concentration was fixed at $10 \mathrm{wt} \%$. The MBA, APS and PEG concentrations were maintained constant at 4, 0.3, and $20 \mathrm{wt} \%$, respectively. All beads were polymerized at $70^{\circ} \mathrm{C}$ for $15 \mathrm{~min}$.

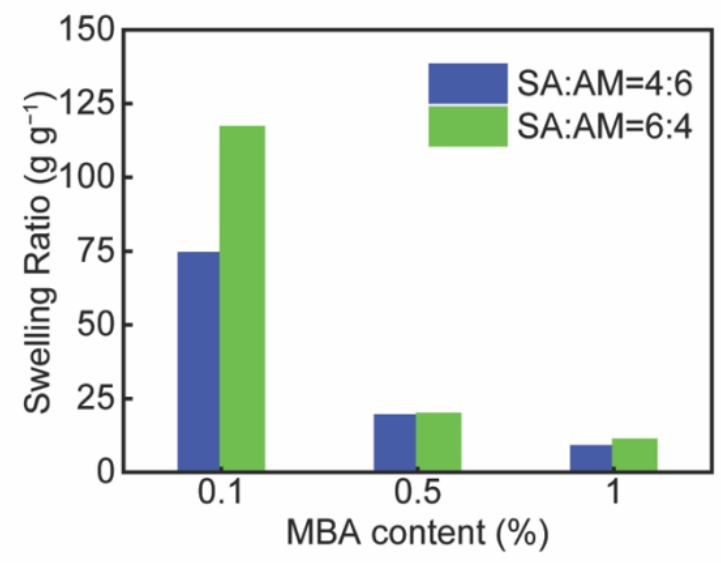

Figure S10. Swelling ratio of the poly(SA-co-AM) beads in DI water. The PAS beads were prepared by reaction mixtures containing 4 or $6 \mathrm{wt} \%$ of SA monomers. The total monomer concentration was fixed at $10 \mathrm{wt} \%$. The APS and PEG concentrations were maintained constant at 0.3 and $10 \mathrm{wt} \%$, respectively. The MBA concentration ranged from 0.1 to $1 \mathrm{wt} \%$. All beads were polymerized at $70^{\circ} \mathrm{C}$ for $15 \mathrm{~min}$. 

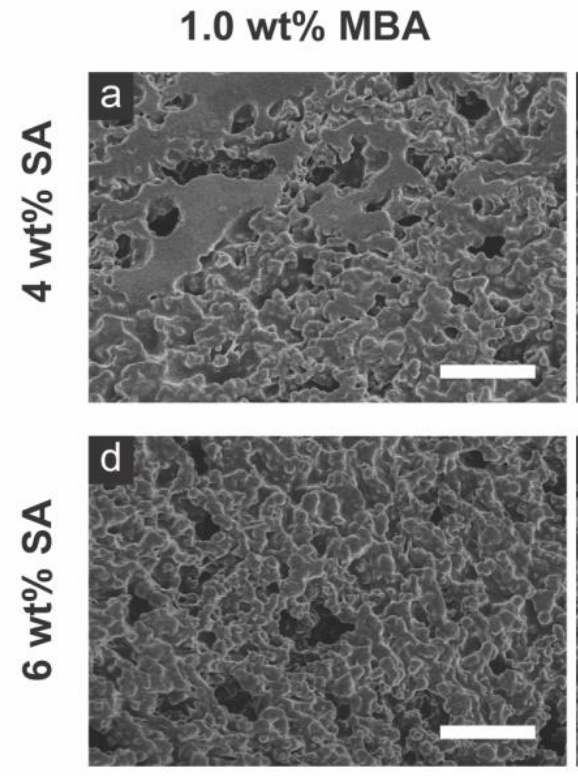

0.5 wt $\%$ MBA

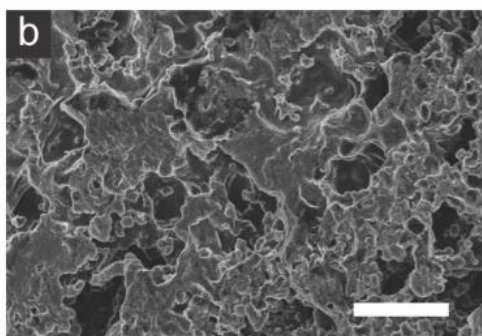

\section{1 wt $\%$ MBA}
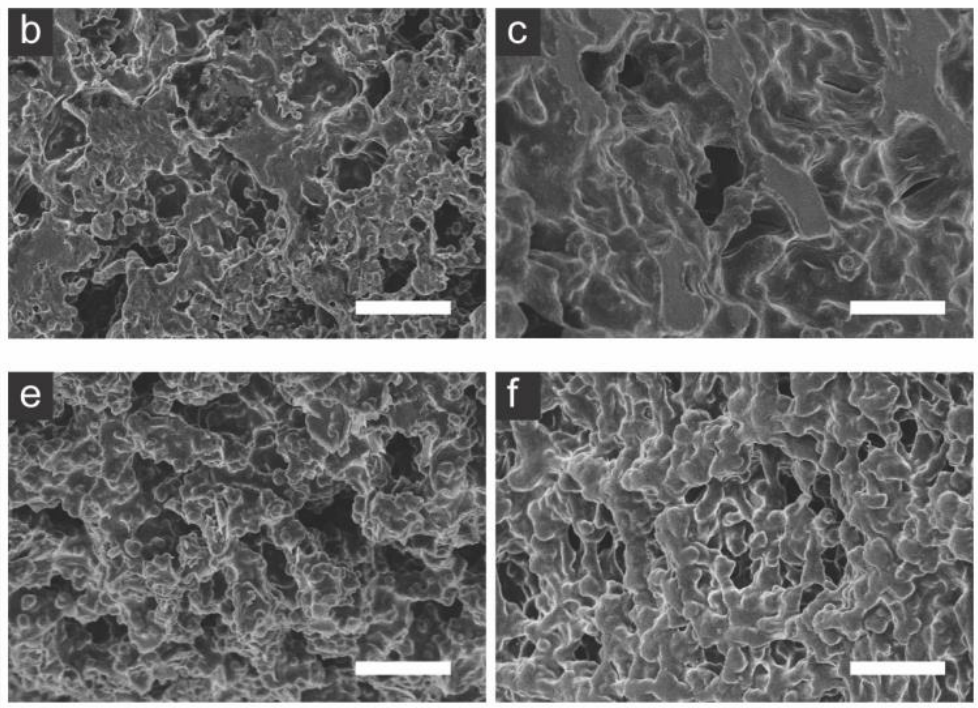

Figure S11. SEM images of the poly(SA-co-AM) beads with different crosslinking degrees and charge densities. The PSAP beads were prepared by reaction mixtures containing 4 or $6 \mathrm{wt} \%$ of SA monomers. The total monomer concentration was fixed at $10 \mathrm{wt} \%$. The APS and PEG concentrations were maintained constant at 0.3 and $10 \mathrm{wt} \%$, respectively. The MBA concentration ranged from 1.0 to $0.1 \mathrm{wt} \%$ : (a, d) $1.0 \mathrm{wt} \%$, (b, e) $0.5 \mathrm{wt} \%$, and (c, f) $0.1 \mathrm{wt} \%$. All beads were polymerized at $70^{\circ} \mathrm{C}$ for $15 \mathrm{~min}$. The scale bars are all $5 \mu \mathrm{m}$. 


\section{Additional Tables}

Table S1. Mercury intrusion test of PSAP beads prepared with different PEG contents

\begin{tabular}{cccc}
\hline $\begin{array}{c}\text { PEG Content } \\
(\mathrm{wt} \%)\end{array}$ & $\begin{array}{c}\text { Total Intrusion Volume } \\
\left(\mathrm{cm}^{3} \mathrm{~g}^{-1}\right)\end{array}$ & $\begin{array}{c}\text { Total Pore Area } \\
\left(\mathrm{m}^{2} \mathrm{~g}^{-1}\right)\end{array}$ & $\begin{array}{c}\text { Bulk Density } \\
\left(\mathrm{g} \mathrm{cm}^{-3}\right)\end{array}$ \\
\hline 2.5 & 0.1395 & 17.017 & 1.0526 \\
5.0 & 0.2398 & 26.125 & 1.0197 \\
7.5 & 0.4871 & 10.871 & 0.8731 \\
10 & 0.6529 & 12.375 & 0.7489 \\
15 & 1.0426 & 14.962 & 0.5730 \\
20 & 1.1126 & 23.179 & 0.5448
\end{tabular}

${ }^{a}$ The mercury intrusion test was operated using the following parameters: the contact angle was $130^{\circ}$, the mercury surface tension was $485 \mathrm{dyn} \mathrm{cm}^{-1}$, and the maximum intrusion pressure was 4.68 psia.

Table S2. The composition of the synthetic urine

\begin{tabular}{cc}
\hline Compound & Concentration $\left(\mathrm{g} \mathrm{L}^{-1}\right)$ \\
\hline $\mathrm{Urea}$ & 15.0075 \\
$\mathrm{NaCl}$ & 2.5715 \\
$\mathrm{Na}_{2} \mathrm{SO}_{4}$ & 2.1305 \\
$\mathrm{KCl}$ & 2.9820 \\
$\mathrm{MgCl}_{2}$ & 0.3809 \\
$\mathrm{NaH}_{2} \mathrm{PO}_{4}$ & 2.3995 \\
$\mathrm{CaCl}_{2}$ & 0.4440 \\
$\mathrm{pH}^{\mathrm{a}}$ & 6.0 \\
\hline
\end{tabular}

The $\mathrm{pH}$ was adjusted by $1 \mathrm{M} \mathrm{NaOH}$ solution. 


\section{References}

(1) Galant, A.; Kaufman, R.; Wilson, J., Glucose: detection and analysis. Food Chemistry 2015, 188, 149-160.

(2) Aebi, H., Catalase. In Methods of enzymatic analysis, Elsevier: 1974; pp 673-684.

(3) Yuan, B.; Pham, M.; Nguyen, T. H., Deposition kinetics of bacteriophage MS2 on a silica surface coated with natural organic matter in a radial stagnation point flow cell. Environmental science \& technology 2008, 42, 7628-7633.

(4) Chen, W.; Jiang, J.; Zhang, W.; Wang, T.; Zhou, J.; Huang, C.-H.; Xie, X., Silver Nanowire-Modified Filter with Controllable Silver Ion Release for Point-of-Use Disinfection. Environmental Science \& Technology 2019, 53, 7504-7512.

(5) Guo, S.; Yao, T.; Ji, X.; Zeng, C.; Wang, C.; Zhang, L., Versatile preparation of nonspherical multiple hydrogel core PAM/PEG emulsions and hierarchical hydrogel microarchitectures. Angewandte Chemie International Edition 2014, 53, 7504-7509.

(6) Guo, S.; Yao, T.; Wang, C.; Zeng, C.; Zhang, L., Preparation of monodispersed porous polyacrylamide microspheres via phase separation in microchannels. Reactive and Functional Polymers 2015, 91, 77-84.

(7) Okay, O.; Sariisik, S. B., Swelling behavior of poly (acrylamide-co-sodium acrylate) hydrogels in aqueous salt solutions: theory versus experiments. European Polymer Journal 2000, 36, 393-399.

(8) Okay, O., General properties of hydrogels. In Hydrogel sensors and actuators, Springer: 2009; pp 1-14. 